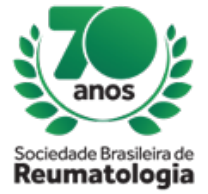

\title{
LUPUS MYOCARDITIS AND SEVERE HEART FAILURE
}

Bruna de Lima Porto (Hospital de Clínicas de Porto Alegre, PORTO ALEGRE, RS, Brasil), Victoria Silveira De Carvalho (Hospital de Clínicas de Porto Alegre, Porto Alegre, RS, Brasil), Ana Laura Fischer Kunzler (Hospital de Clínicas de Porto Alegre, Porto Alegre, RS, Brasil), Maurício Simoni Candaten (Hospital de Clínicas de Porto Alegre, Porto Alegre, RS, Brasil), Afonso Papke (Hospital de Clínicas de Porto Alegre, Porto Alegre, RS, Brasil), Micheline Sulzbacher Batista (Hospital de Clínicas de Porto Alegre, Porto Alegre, RS, Brasil), Andrese Aline Gasparin (Hospital de Clínicas de Porto Alegre, Porto Alegre, RS, Brasil), Ricardo Machado Xavier (Hospital de Clínicas de Porto Alegre, Porto Alegre, RS, Brasil)

\section{BACKGROUND}

Myocarditis is an often asymptomatic manifestation of heart involvement in the course of systemic lupus erythematosus (SLE), with a prevalence of 8 to 25 percent in past studies. Typical clinical features include chest pain with tachycardia, palpitations and/or symptoms of heart failure. Echocardiogram may assess the extent of ventricular dysfunction (systolic and/or diastolic) and hemodynamic compromise as low ejection fraction is detected in the majority of the patients. The gold standard for diagnosis confirmation remains endomyocardial biopsy. However, this procedure is not routinely used because of its low sensitivity and potential complications. Thus, myocarditis diagnosis is often achieved using clinical findings, biological markers, and imaging, classically echocardiography.

\section{CASE REPORT}

A previously healthy 40-year-old African-descendant female patient presented the first medical appointment in the service of rheumatology, with a five-month history of erythematous skin lesions, photosensitivity, alopecia, arthralgia, elevation of pressure levels, progressive fatigue and dyspnea. Physical examination showed signs of pulmonary congestion and patient was admitted for etiological management and investigation. Complementary exams showed cardiomegaly, low complements, pleural and pericardial effusions, antinuclear factor (ANA) 1: 1280 homogeneous nuclear pattern and Anti-dsDNA $1 / 320$, which, in adittion to the patient's clinical condition, confirmed the diagnosis of SLE and suggested the presence of nephritis (loss of renal function, nephrotic proteinuria, active urinary sediment) and myocarditis (echocardiogram with severe diffuse hypokinesia and ejection fraction of $27 \%$ ). Due to the severity of the heart failure, she was transferred to the intensive care unit (ICU) where she received parenteral vasodilators, rigorous blood pressure control, and a 3-day steroid pulse for treatment of nephritis and myocarditis, developing progressive clinical improvement, receiving discharge from the ICU 5 days later. Diuretic, vasodilator and antihypertensive drugs were passive of gradual reduction on subsequent days followed by a control echocardiogram performed 1 week after pulse therapy evidencing preserved left ventricular and global systolic function and ejection fraction of $54 \%$. During hospitalization, she started a cyclophosphamide program and was discharged with prednisone $1 \mathrm{mg} / \mathrm{kg} / \mathrm{day}$ with an outpatient return plan at 1 month for reassessment and follow-up.

\section{CONCLUSION}

Despite the low incidence of HF due to lupus myocarditis, this condition requires high clinical suspicion due to high morbidity and mortality. Improvement in systolic function has been noted in some patients treated with glucocorticoids, other immunosuppressants (e.g., cyclophosphamide, azathioprine), or intravenous immune globulin. A favorable outcome depends on the rapid recognition of this condition and appropriate treatment. 